\title{
CMEARTICLE
}

\section{Clinics in diagnostic imaging (198)}

Yong Quan Alvin Soon ${ }^{1}$, MBBS, FRCR, Hsien Min Low ${ }^{1}$, MBBS, FRCR, Cheong Wei Terence Huey $^{2}$, MBBS, FRCSEd, Gervais Khin-Lin Wansaicheong ${ }^{1}$, MBBS, FRCR
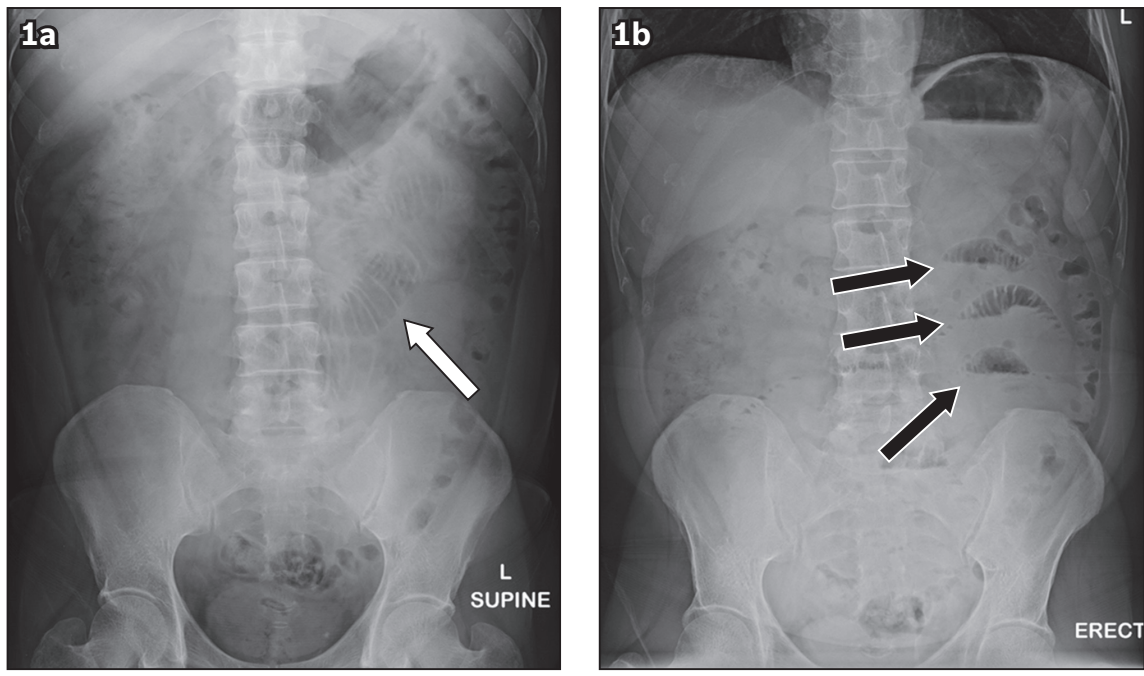

Fig. 1 (a) Supine and (b) erect abdominal radiographs.
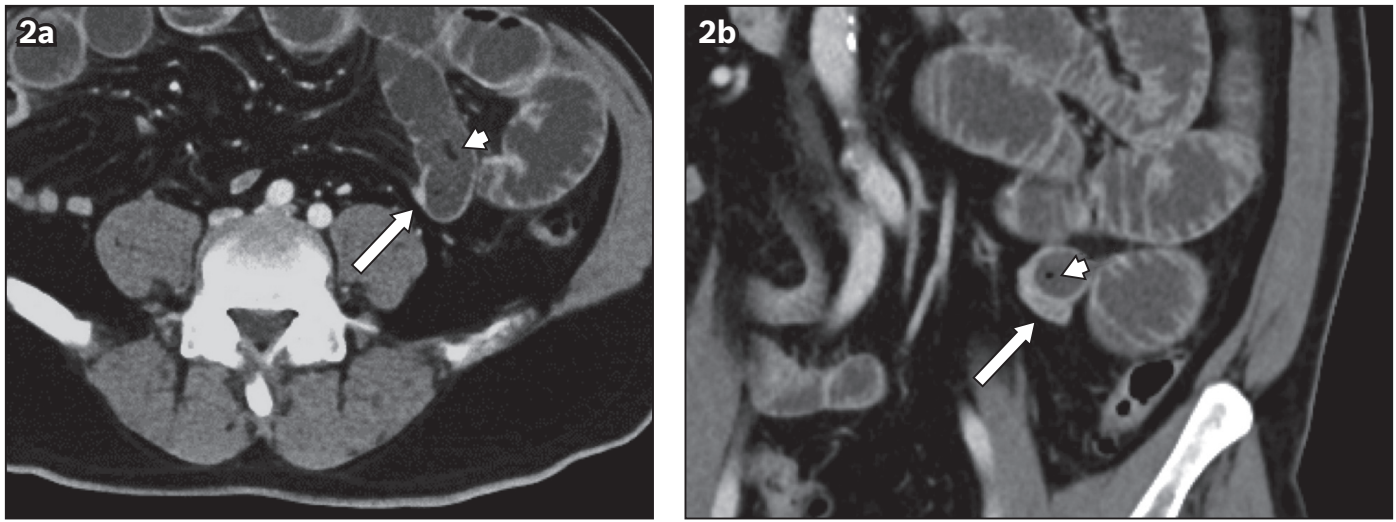

Fig. 2 (a) Axial and (b) coronal CT images of the abdomen and pelvis in the portal venous phase.

\section{CASE PRESENTATION}

A 60-year-old Chinese man with a past medical history of hypertension, hyperlipidaemia and previous cerebrovascular accident six years ago presented with a one-day history of acute abdominal pain and vomiting. This was associated with abdominal distension and symptoms of obstipation. He did not complain of any constitutional symptoms such as weight loss or loss of appetite. He did not report having any prior abdominal surgery.
Physical examination revealed mild, central abdominal tenderness. The abdomen was distended, but no guarding or rebound tenderness was elicited. No mass or lump was felt in the hernial orifices of the groin.

Abdominal radiography (Fig. 1) and contrast-enhanced computed tomography (CT) of the abdomen and pelvis (Fig. 2) were performed. What do these images show and what is the diagnosis? 


\section{IMAGE INTERPRETATION}

Abdominal radiography shows a few dilated small bowel loops on the supine view (arrow, Fig. 1a). Several air-fluid levels are also noted on the erect abdominal radiograph (arrows, Fig. 1b). These findings are in keeping with the submitted history of intestinal obstruction. No radiographic evidence of pneumoperitoneum is seen.

$\mathrm{CT}$ confirmed the presence of multiple dilated small bowel loops. A distinct transition point is seen in the left lower quadrant, where the dilated small bowel loops abruptly return to normal calibre (arrowheads, Fig. 2). The bowel walls at the point of transition do not show any mural thickening and no extrinsic mass is seen that could potentially be causing obstruction. Of note, a few foci of fat density tissue are seen just proximal to the transition point (arrows, Fig. 2).

\section{DIAGNOSIS}

Small bowel obstruction secondary to a bezoar.

\section{CLINICAL COURSE}

The patient was scheduled for urgent surgery in view of the CT findings of small bowel obstruction. He underwent a diagnostic laparoscopy, which confirmed the presence of small bowel obstruction with a discrete transition point. Distal to the transition point, the small bowel loops were collapsed. The bowel was otherwise healthy and viable. The cause of the obstruction was due to a hard intraluminal mass, presumably a bezoar. As such, the affected small bowel was exteriorised and an enterotomy made to remove the bezoar, with subsequent closure of the enterotomy. The extracted bezoar visually resembled a palm seed from Nypa fruticans, also known colloquially as an attap chee (Fig. 3).

An on-table oesophagogastroduodenoscopy was also performed. It showed a periampullary diverticulum containing a bezoar that was subsequently retrieved endoscopically. On extraction, the bezoar within the periampullary diverticulum resembled a mushroom (Fig. 4a) that, in retrospect, could be seen on the preoperative CT image (Fig. 4b). No bezoar was seen in the stomach. The patient's post-surgery recovery was uneventful, with no further complaints on follow-up. On further questioning postoperatively, the patient reported that he had lost his dentures recently and had swallowed an attap chee whole without chewing.

\section{DISCUSSION}

Small bowel intestinal obstruction is a common surgical condition encountered in clinical practice. In patients who had previous abdominal surgery, the commonest cause of small bowel obstruction is adhesions. In patients with no prior history of abdominal surgery (i.e. the virgin abdomen), causes of intestinal obstruction are varied and can be classified as intraluminal, mural or extramural aetiologies (Box 1).

Bezoars are a well recognised, albeit uncommon, cause of small bowel obstruction, contributing to $4 \%$ of cases. ${ }^{(1,2)}$ They are masses composed of partially digested or undigested food material within the lumen of the gastrointestinal tract and are formed due to high fibre intake, inadequate mastication and reduced gastric motility. Hence, bezoars are prevalent among patients with delayed gastric emptying, such as after a vagotomy or gastrectomy, or as a result of autonomic dysfunction from diabetes mellitus. ${ }^{(3,4)}$

Bezoars are commonly seen in the stomach and the small bowel. Although gastric bezoars are more common, small bowel bezoars are more likely to present with intestinal obstruction. Most small bowel bezoars are located in the ileum, approximately $50-70 \mathrm{~cm}$ proximal from the ileocaecal junction..$^{(5-7)}$ It has been

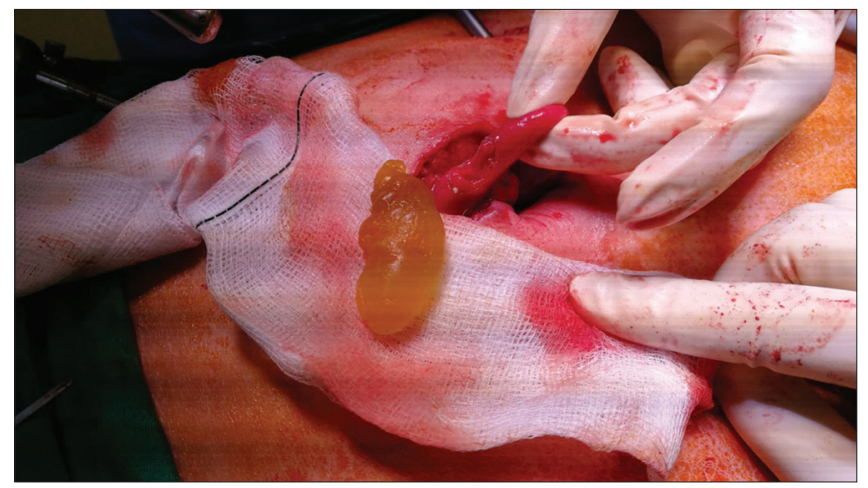

Fig. 3 Intraoperative photograph shows an ovoid bezoar resembling the endosperm of Nypa fruticans at the site of the obstruction.
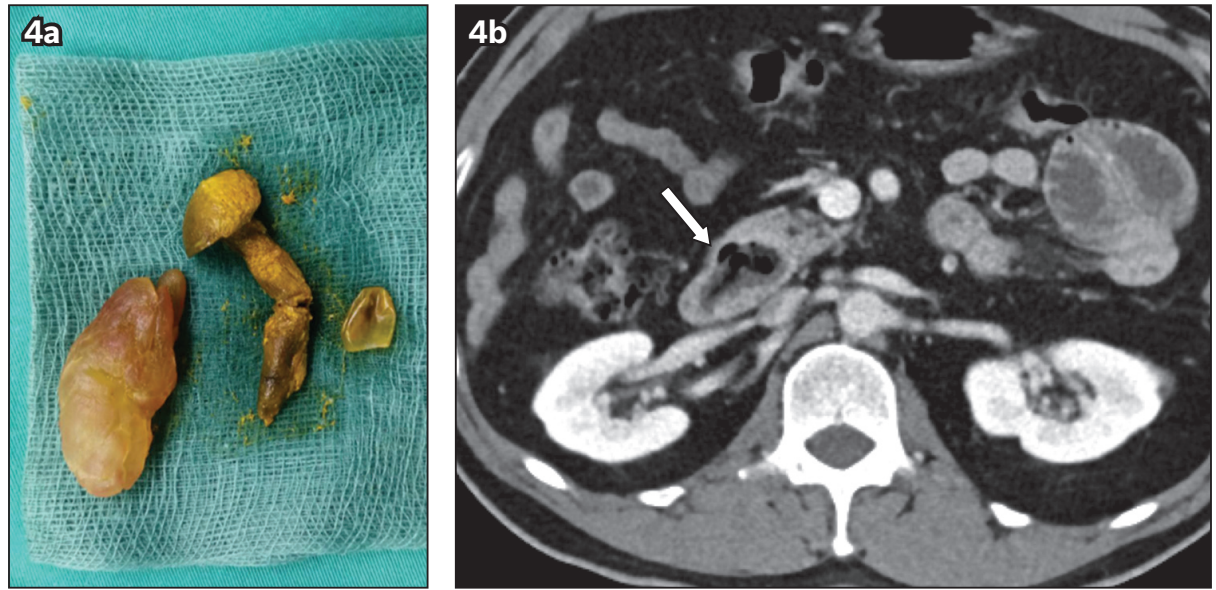

Fig. 4 (a) Photograph shows the probable attap chee bezoar (left) and the extracted mushroom (right). (b) Axial CT image shows the mushroom phytobezoar in the periampullary diverticulum (arrow). 


Box 1. Causes of virgin abdomen intestinal obstruction:
Intraluminal
- Gallstone ileus
- Food bolus obstruction
- Foreign body/bezoar
- Roundworm mass
Mural
- Strictures
- Crohn's disease
- Carcinomas
Extramural
- Volvulus
- Intussusception
- Obstructed hernia
- Congenital bands

postulated that this is due to the smaller luminal calibre of the ileum, reduced bowel motility and the fact that a large amount of water reabsorption occurs in the small bowel. Water reabsorption causes hardening of the bezoar, resulting in impaction and obstruction. A few different types of bezoars have been described, with phytobezoars being the most common. ${ }^{(8,9)}$ Other rarer forms of bezoars include trichobezoars, which are seen in psychiatric patients who ingest hair ${ }^{(10)}$ and lactobezoars, which are masses of milk coagulum found in infants. ${ }^{(11)}$

The clinical presentation of small bowel obstruction due to a bezoar is similar to any other case of small bowel obstruction. Patients present with colicky abdominal pain, vomiting, abdominal distension and constipation. ${ }^{(12)}$ On conventional abdominal radiography, dilated small bowel loops and multiple air-fluid levels are seen. In the appropriate clinical context, this is in keeping with small bowel obstruction. The offending bezoar is usually not detected on radiography unless it is large.

Contrast-enhanced CT of the abdomen and pelvis is the imaging modality of choice in the evaluation of patients with intestinal obstruction. CT allows the radiologist to identify the site of obstruction (commonly referred to as the transition point) and, more importantly, elucidate the cause of obstruction. CT also helps to evaluate for the presence of complications such as perforation or bowel ischaemia.

The typical CT appearance of a bezoar is a discrete intraluminal mass that contains multiple gas locules in its interstices and is located at the transition point where it is causing intestinal obstruction (Figs. 5 \& 6). Primary small bowel bezoars are uncommon and tend to occur in patients with significant small bowel stasis, such as in Crohn's disease, tuberculosis, small bowel tumour and diverticulosis. Gastric bezoars are often seen together with small bowel bezoars and may migrate distally, eventually resulting in small bowel obstruction. ${ }^{(5)}$ In our patient, no gastric bezoar was detected on CT and subsequent oesophagogastroduodenoscopy. However, a bezoar was found in a periampullary diverticulum, which could be seen on CT retrospectively.

An entity that can mimic the appearance of a bezoar is the 'small bowel faeces' sign (Fig. 7). This imaging sign is not
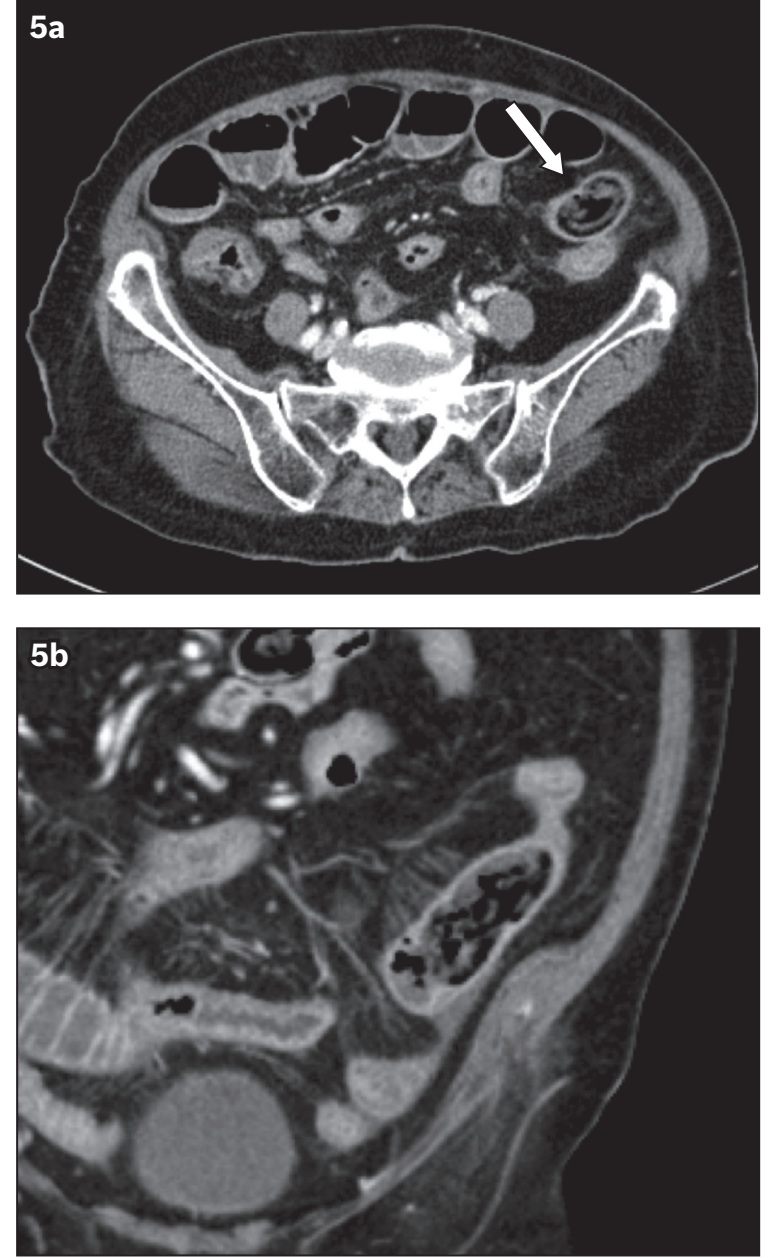

Fig. 5 A 60-year-old male patient presented with intestinal obstruction. (a) Axial and (b) coronal CT images show a well-defined mass lesion (arrow) with a mottled appearance due to the presence of multiple gas locules trapped in its interstices. The patient underwent surgery, which showed a phytobezoar composed of undigested vegetable material.

specific and may be seen in any case of high-grade small bowel obstruction. This sign is due to the stasis of intraluminal contents. Due to the stasis, water reabsorption occurs from the luminal contents, resulting in the formation of particulate material mixed with gas bubbles, which resembles faeces. This is also reflected on CT images, on which a long segment of dilated small bowel containing mottled gas locules is observed, reminiscent of faecal material. ${ }^{(13)}$

Distinguishing intraluminal bezoars from the 'small bowel faeces' sign is a challenge with many overlapping features on CT. Indeed, in a study by Delabrousse et al, several CT features that were thought to be exclusively seen in bezoars were also detected in cases that had the 'small bowel faeces' sign. Nonetheless, their study showed that features such as a well-encapsulated wall, a similar bezoar-like lesion in the stomach and the 'floating fat density' sign were useful in distinguishing cases of bezoars from 'small bowel faeces'. ${ }^{(13)}$ In our case, some foci of fat density were also seen proximal to the obstructing lesion (Figs. $8 \& 9$ ). This is suggestive of the aforementioned 'floating fat density sign', which may be seen in cases of bezoar-induced small bowel obstruction. ${ }^{(13)}$ This sign refers to the presence of fat-density foci with an attenuation ranging between -50 and -150 Hounsfield 

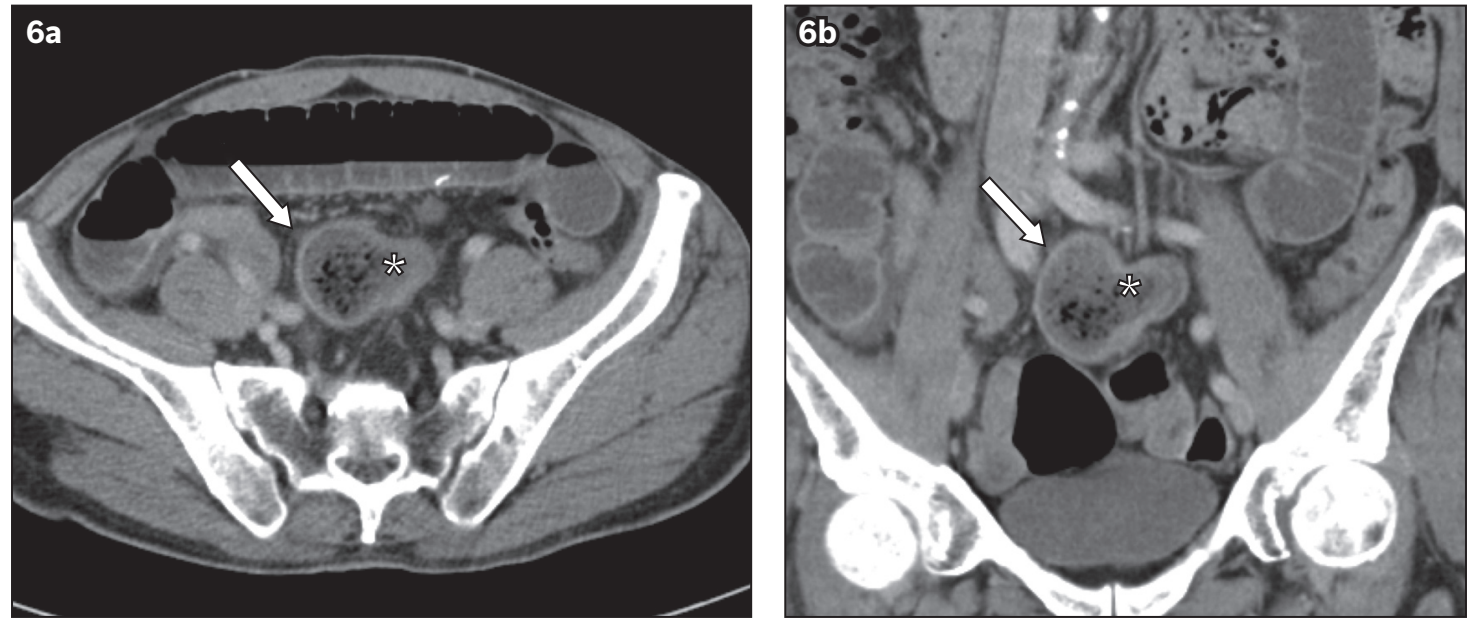

Fig. 6 A 83-year-old female patient who presented with intestinal obstruction. (a) Axial and (b) coronal CT images show a well-defined mass lesion (arrows) with a heterogeneous mottled appearance due to gas locules trapped within its interstices, in keeping with a bezoar. The bezoar has an unusual T-shaped appearance, as part of it was trapped within a Meckel's diverticulum (asterisk). The obstructing bezoar was surgically removed and shown to be a phytobezoar.

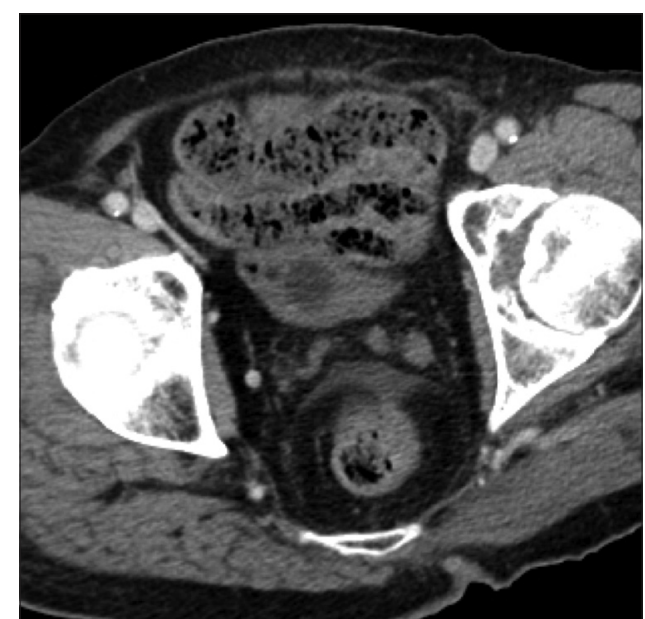

Fig. 7 Axial CT image shows 'small bowel faeces' sign in a 75-year-old woman. Note that the appearance of the small bowel contents is very similar to the bezoars seen in Figs. 5 and 6. As such, the 'small bowel faeces' sign can easily be mistaken for a bezoar and vice versa.

units, seen proximal to the bezoar. Delabrousse et al postulate that this appearance is due to the sum of the attenuation of softtissue debris as well as the negative attenuation of gas locules. ${ }^{(13)}$

Our case is unique, as the bezoar that was extracted resembled an attap chee. Furthermore, the classic appearance of a bezoar was not found on CT. An ovoid low-density structure was seen on $\mathrm{CT}$, instead of the usual gas-mottled appearance of bezoars.

CT images of off-the-shelf seeds of Nypa fruticans showed that the seeds were hyperdense (Fig. 10). This appearance was inconsistent with the findings of our patient, where the seed was nearly isodense to the surrounding fluid within the bowel lumen. We postulate that the low density of the seed in our patient could be due to its partial digestion as it traversed the digestive tract. This highlights the difficulty in diagnosing unusual bezoars as the cause of small bowel obstruction.

The definitive management of small bowel dilatation with a transition point in a virgin abdomen is often surgical. In our case, the initial working diagnosis was likely a congenital adhesion

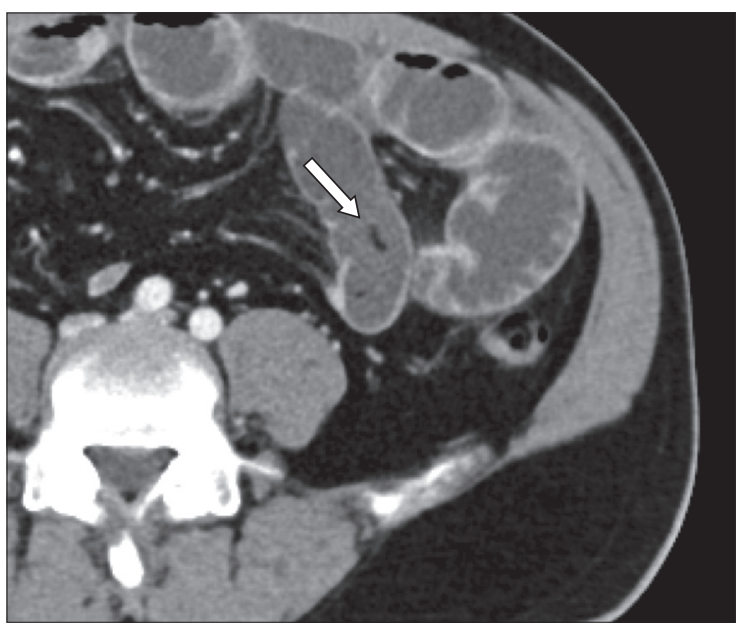

Fig. 8 Selected axial CT images of the patient in Fig. 1 show the 'floating fat density' sign (arrow) just proximal to the obstructing bezoar.

band, as it was not very evident to the surgeon that there was a bezoar on the images. However, on laparoscopy, a hard intraluminal bezoar mass was noted proximal to the transition point. As the calibre of the bowel tends to narrow as it progresses towards the caecum, it is unlikely for peristalsis alone to push the bezoar through to the more capacious colon. Surgical intervention aims to remove the bezoar as shown in this case or, in a softer phytobezoar, to manually break it intraluminally and milk it into the caecum, avoiding the need for an enterotomy. Surgery also allows an assessment of the proximal bowel to ensure that there are no other similar offending bezoars, such as another seed in a more proximal location, and to exclude other bezoars in the stomach, which could cause repeated problems.

In this clinical case, it is likely that the patient developed a phytobezoar from inadequate mastication, resulting in him swallowing the attap chee endosperm whole. Poor mastication is often seen in elderly patients with poor dentition as well as patients with neurological disorders (e.g. stroke) and results in large food particles being swallowed. ${ }^{(14)}$ While studies have shown 

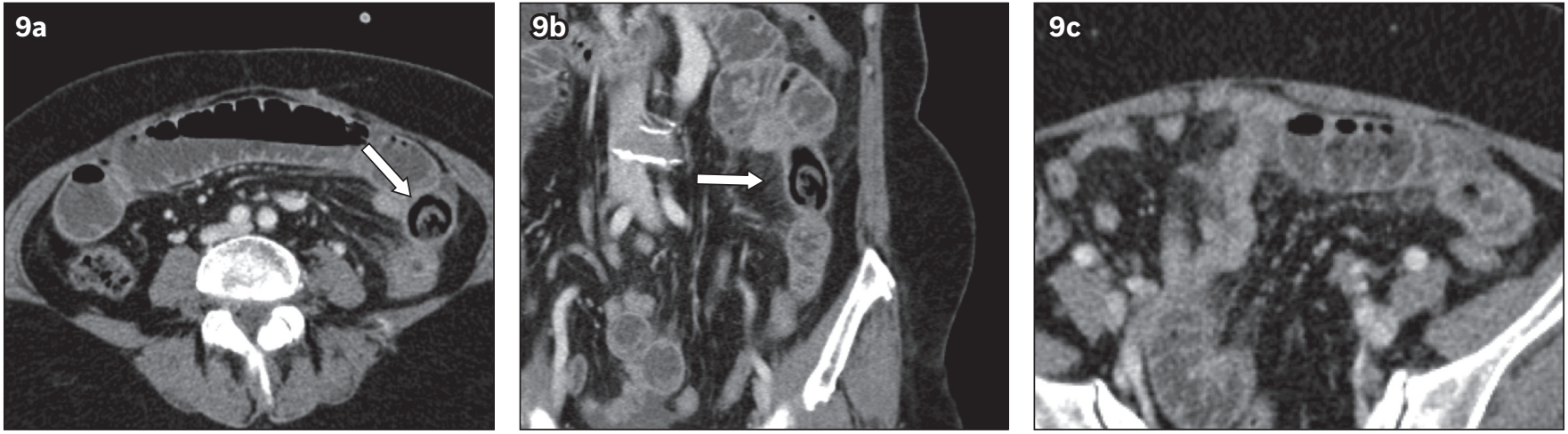

Fig. 9 A 67-year-old man presented with intestinal obstruction. (a) Axial and (b) coronal CT images show an obstructing bezoar (arrows) with a mottled appearance due to gas locules, proven on surgery to be a phytobezoar resembling a shiitake mushroom. (c) Axial CT image of the abdomen shows the presence of several small foci of fat-density material just proximal to the phytobezoar, which likely correspond to the 'floating fat density' sign.

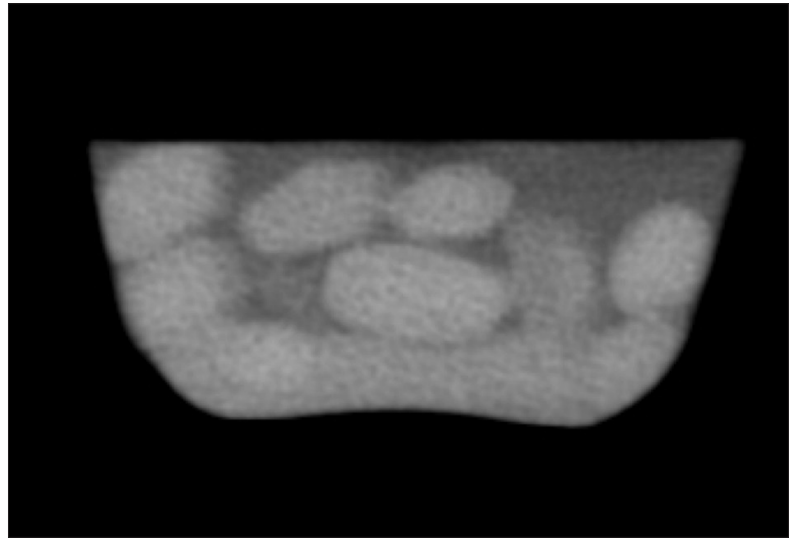

Fig. 10 CT image of commercially available Nypa fruticans seeds shows them to be hyperdense with an attenuation of approximately 50-60 Hounsfield units.

that poor dentition and mastication are risk factors for bezoar formation, ${ }^{(15)}$ there is currently no published study available, to our knowledge, that correlates food particle size and the incidence of bezoar formation.

In conclusion, bezoars are an unusual cause of small bowel obstruction. Even bezoars with a classic appearance may be mistaken for the 'small bowel faeces' sign in longstanding highgrade small bowel obstructions. In the absence of the classic gas-mottled appearance of phytobezoars, the diagnosis can be challenging, as in this case.

\section{REFERENCES}

1. Razavianzadeh N, Foroutan B, Honarvar F, Forozeshfard M. Small bowel obstruction attributable to phytobezoar. Oxf Med Case Reports 2016 ; 2016:omw092.

2. Ho TW, Koh DC. Small-bowel obstruction secondary to bezoar impaction: a diagnostic dilemma. World J Surg 2007; 31:1072-80.

3. Dikicier E, Altintoprak F, Ozkan OV, Yagmurkaya O, Uzunoglu MY. Intestinal obstruction due to phytobezoars: an update. World J Clin Cases 2015; 3:721-6.
ABSTRACT A 60-year-old man presented with abdominal pain. He was later diagnosed on imaging to have high-grade small bowel obstruction. The patient underwent surgery, and a hard, rounded bezoar resembling the endosperm of Nypa fruticans, colloquially known as attap chee, was found at the point of obstruction. Small bowel obstruction is a common acute surgical condition with multiple causes, including bezoars. We discuss the typical imaging features of bezoars causing small bowel obstruction as well as potential pitfalls that can mimic the appearance of a bezoar.

Keywords: bezoar, floating fat-density sign, phytobezoar, small bowel faeces sign, small bowel obstruction

4. Yildirim T, Yildirim S, Barutcu O, Oguzkurt L, Noyan T. Small bowel obstruction due to phytobezoar: CT diagnosis. Eur Radiol 2002; 12:2659-61.

5. Kement M, Ozlem N, Colak E, et al. Synergistic effect of multiple predisposing risk factors on the development of bezoars. World J Gastroenterol 2012;18:960-4.

6. Oh SH, Namgung H, Park MH, Park DG. Bezoar-induced small bowel obstruction. J Korean Soc Coloproctol 2012; 28:89-93.

7. Wang PY, Wang X, Zhang $L$, et al. Bezoar-induced small bowel obstruction: clinical characteristics and diagnostic value of multi-slice spiral computed tomography. World J Gastroenterol 2015; 21:9774-84.

8. Yakan S, Sirinocak A, Telciler KE, Tekeli MT, Deneçli AG. A rare cause of acute abdomen: small bowel obstruction due to phytobezoar. Ulus Travma Acil Cerrahi Derg 2010; 16:459-63.

9. Sheikh AB, Akhtar A, Nasrullah A, Haq S, Ghazanfar H. Role of laparoscopy in the management of acute surgical abdomen secondary to phytobezoars. Cureus 2017; 9:e1363.

10. Lalith S, Gopalakrishnan KL, Ilangovan G, Jayajothi A. Rapunzel Syndrome. J Clin Diagn Res 2017; 11:TD01-2.

11. Heinz-Erian P, Gassner I, Klein-Franke A, et al. Gastric lactobezoar - a rare disorder? Orphanet J Rare Dis 2012; 7:3.

12. Erzurumlu K, Malazgirt Z, Bektas A, et al. Gastrointestinal bezoars: a retrospective analysis of 34 cases. World J Gastroenterol 2005; 11:1813-7.

13. Delabrousse E, Lubrano J, Sailley N, et al. Small-bowel bezoar versus smallbowel feces: CT evaluation. AJR Am J Roentgenol 2008; 191:1465-8.

14. van der Bilt A. Assessment of mastication with implications for oral rehabilitation: a review. J Oral Rehabil 2011; 38:754-80.

15. Escamilla C, Robles-Campos R, Parrilla-Paricio $P$, et al. Intestinal obstruction and bezoars. J Am Coll Surg 1994; 179:285-8. 


\section{SINGAPORE MEDICAL COUNCIL CATEGORY 3B CME PROGRAMME}

\section{(Code SMJ 201908B)}

Question 1. Regarding bezoars:

(a) Small bowel bezoars are more common than gastric bezoars.

(b) Small bowel bezoars are more likely to present with bowel obstruction than gastric bezoars.

(c) The most common site of bowel obstruction due to bezoar is the ileocaecal valve.

(d) The most common type of bezoar is the trichobezoar.

Question 2. Predisposing factors for bezoar formation include:

(a) High fibre intake.

(b) Previous colectomy.

(c) Previous vagotomy.

(d) Previous gastrectomy.

Question 3. Regarding the imaging evaluation of small bowel obstruction:

(a) Abdominal radiography is sufficient for evaluating the cause of small bowel obstruction.

(b) Computed tomography $(\mathrm{CT})$ is often diagnostic in identifying bezoars as a cause of intestinal obstruction if the typical imaging features are present.

(c) CT is useful to identify the site of obstruction.

(d) CT is useful to identify the complications of obstruction (perforation or bowel ischaemia).

Question 4. Regarding the CT imaging finding of phytobezoar:

(a) It is a lobulated mass arising from the wall of the bowel.

(b) It is a discrete intraluminal mass with gas locules in the interstices.

(c) It is usually identified at the transition point of dilated bowel loops.

(d) It may be misdiagnosed as the 'small bowel faeces' sign.

Question 5. Regarding the management of bezoars:

(a) The definitive management is often surgical.

(b) Enterotomy is always necessary to remove the obstructing bezoar.

(c) Conservative management with intravenous hydration and nasogastric tube decompression is sufficient.

(d) Assessment of the proximal bowel loops intraoperatively is recommended to exclude a concomitant bezoar in a more proximal location.

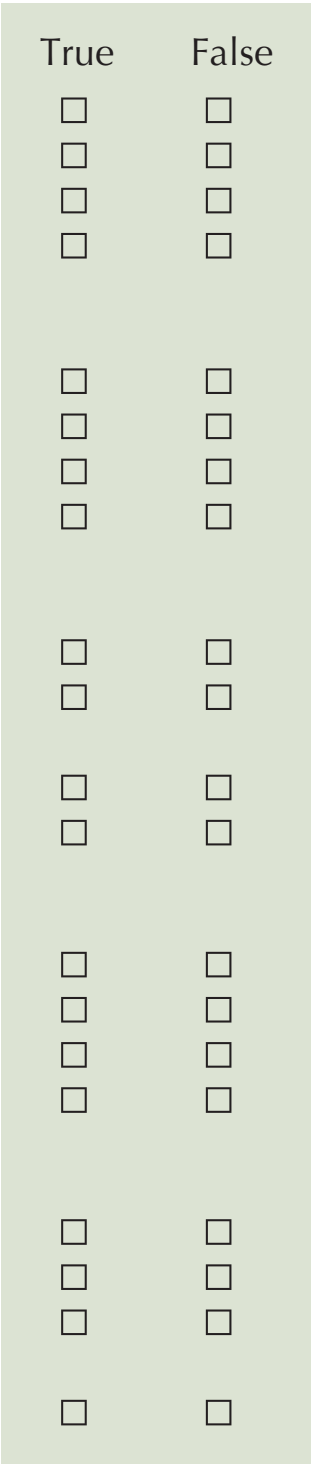

\section{Doctor's particulars:}

Name in full:

MCR no.:

Specialty:

Email:

\footnotetext{
SUBMISSION INSTRUCTIONS:

Visit the SMJ website: http://www.smj.org.sg/current-issue and select the appropriate quiz. You will be redirected to the SMA login page.

For SMA member: (1) Log in with your username and password (if you do not know your password, please click on 'Forgot your password?'). (2) Select your answers for each quiz and click 'Submit'.

For non-SMA member: (1) Create an SMJ CME account, or log in with your SMJ CME username and password (for returning users). (2) Make payment of SGD 21.40 (inclusive of $7 \%$ GST) via PayPal to access this month's quizzes. (3) Select your answers for each quiz and click 'Submit'.

RESULTS:

(1) Answers will be published online in the SMJ October 2019 issue. (2) The MCR numbers of successful candidates will be posted online at the SMJ website by 10 October 2019. (3) Passing mark is $60 \%$. No mark will be deducted for incorrect answers. (4) The SMJ editorial office will submit the list of successful candidates to the Singapore Medical Council. (5) One CME point is awarded for successful candidates. (6) SMC credits CME points according to the month of publication of the CME article (i.e. points awarded for a quiz published in the December 2017 issue will be credited for the month of December 2017, even if the deadline is in January 2018)

Deadline for submission (August 2019 SMJ 3B CME programme): 12 noon, 3 October 2019.
} 